Pediatrics

\title{
"Your own pace, your own path": perspectives of adolescents navigating life after bariatric surgery
}

\author{
Ming K. Li $\mathbb{D}^{1}$, Thrmiga Sathiyamoorthy ${ }^{1}$, Andrea Regina ${ }^{2,3}$, Michele Strom ${ }^{3}$, Alene Toulany $y^{1,4}$ and Jill Hamilton $\mathbb{D}^{1,3} 3^{凶}$ \\ (c) The Author(s), under exclusive licence to Springer Nature Limited 2021
}

INTRODUCTION: Bariatric surgery, an established weight-loss tool, may be offered to some adolescents with severe obesity. However, few studies explore adolescents' postoperative experiences beyond physical and metabolic outcomes and quality-of-life measures.

METHODS: Between 2016 and 2021, 45 semi-structured interviews were conducted with adolescents (16-20 years) at 6 months $(N=15), 12$ months $(N=15)$, and 24 months $(N=15)$ following bariatric surgery. A deductive thematic analysis framework was applied by two independent coders (Cronbach's $a=0.84$ ). Themes were identified and refined iteratively, and discrepancies were resolved through discussion.

RESULTS: Five major themes emerged related to: (1) weight-loss expectations vs. reality, (2) social landscape, (3) body image, (4) eating and moving, and (5) challenges for long-term success. The pace of and satisfaction with weight loss and side effects was heterogenous among participants, with most changes occurring early and stabilizing by 24 months. Adolescents adapted over time to their new social landscapes (e.g., relationships) and reported improved body image and confidence, yet persistently struggled to reconcile their internal identity with evolving external perceptions and discomfort with new attention. Participants experienced changes to lifestyle routines after surgery (e.g., eating, moving, habits), which introduced distress at 6 months but resolved over time. Life transitions in early adulthood (e.g., moving away, university/college, employment), concurrent with their evolving and increasing autonomy, physical, social, and financial independence, imposed unexpected challenges to postoperative routines and support systems. Participants unanimously reaffirmed that bariatric surgery is a lifelong journey and that they were committed to long-term success.

CONCLUSION: Our findings provide insight into optimizing adolescent selection for bariatric surgery and perioperative support. Specifically, important life transitions during this developmental period impact postoperative experiences and outcomes and adolescents may benefit from postoperative counseling focused on managing weight-loss expectations and adapting to evolving nutritional needs and changing social circumstances.

International Journal of Obesity (2021) 45:2546-2553; https://doi.org/10.1038/s41366-021-00928-w

\section{INTRODUCTION}

Bariatric surgery has been well established as the most effective treatment option for sustained weight loss in adults [1]. While healthy lifestyle modifications remain the mainstay of obesity management approaches for pediatric patients, there is growing evidence supporting the safety and efficacy of bariatric surgery for adolescents with severe obesity [1-4]. Recent studies have documented approximately $30 \%$ weight loss and remission of hypertension and type 2 diabetes mellitus in adolescents, although factors facilitating long-term sustained weight reduction have not yet been elucidated [5-7].

The transition from adolescence to early adulthood is a period of development characterized by physiologic, psychologic, and social changes which present unique challenges [8, 9]. The transition from pediatric, parent-supervised healthcare to more independent, patient-centred adult healthcare is a high-risk period for youth with chronic health conditions marked by increased rates of loss to follow-up, disengagement, and other barriers to transition of care $[10,11]$. Thus, it is imperative to understand post-bariatric surgery experience in this patient population to address their complex needs and reduce risk of chronic morbidity in adulthood.

Much of the literature in this emerging field focuses on physical and metabolic outcomes, with some studies measuring postoperative quality of life (QOL) [12-16]. There are only a few qualitative studies exploring postoperative outcomes through interviews, often cross-sectional in nature or limited in focus to specific outcomes, with shorter follow-up timeframes and in

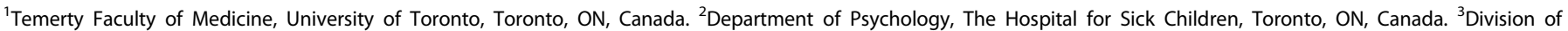

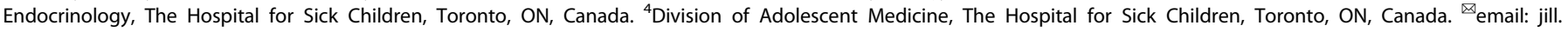
hamilton@sickkids.ca
} 
different healthcare systems [13, 17-19]. Although studies have demonstrated substantial and durable bodyweight reduction and cardiometabolic benefits for adolescent patients, follow-up findings vary based on patient trajectories $[20,21]$. One study demonstrated rapid weight loss and improved weight-related QOL across postoperative year 1, followed by stabilization and modest weight regain, as well as decline in weight-related QOL [16]. Recent reviews reported peak QOL improvement at 6 or 12 months, but noted the paucity of long-term data on other psychological and social outcomes [12, 15].

Our group assessed preoperative perspectives of prospective adolescent bariatric surgery patients in a previous study and were interested in following these participants postoperatively to evaluate how surgery has met or differed from their expectations [22]. To the best of our knowledge, there is no longitudinal study tracking qualitative experiences of adolescents at various timepoints following bariatric surgery. Thus, we aimed to longitudinally explore adolescent patients' postoperative journeys at 6,12 , and 24 months following bariatric surgery.

\section{METHODS}

Our research team included an endocrinologist and clinician-investigator $(\mathrm{JH})$, a clinical psychologist experienced in working with adolescent bariatric surgery patients (AR), an adolescent medicine specialist (AT), as well as a STOMP research coordinator (MS) and research assistants (ML and TS). All team members are experienced in qualitative research $[22,23]$.

\section{Patient population}

The Hospital for Sick Children (SickKids) is the largest academic pediatric tertiary referral center in Canada, and was the only center offering bariatric surgery for adolescents in Canada until 2019. From January 2009 to June 2020, 59 bariatric surgery procedures have been performed at our center by a pediatric surgeon in conjunction with a high-volume adult bariatric surgeon, conforming to best practice guidelines [1].

All bariatric surgery candidates are identified from the SickKids Team Obesity Management Program (STOMP), a 2-year program with behavioral modification to encourage healthy lifestyle choices through individual counseling and group sessions. Patients who express interest in surgery are assessed by the interdisciplinary STOMP team and referred for surgery based on guideline-recommended criteria [1]. Our center offers laparoscopic vertical sleeve gastrectomy (VSG) and Roux-en-Y gastric bypass (RYGB) procedures, and patients choose their preferred procedure, unless medically contraindicated.

Preoperative preparation involves psychosocial assessments with a psychologist and education sessions with a nurse/nurse practitioner, dietitian, and exercise counselor regarding pre- and postoperative routines. Candidates attend individual and group coaching sessions, including a bariatric surgery support group, which connects candidates with postoperative patients to understand their experiences.

Following surgery, patients are followed in the program for 2 years. Follow-up care includes a postoperative surgery support group, individual assessments with the interdisciplinary care team, and regular medical follow-up. Patients are then transitioned to primary care providers with access to dietary support. A practical reference guide was developed by our provincial network of adult bariatric programs to assist with management of bariatric surgery patients in primary care [24].

\section{Patient recruitment}

All English-speaking STOMP patients who underwent bariatric surgery between 2016 and 2020 were eligible. In total, 26 patients were approached by our research team 2-4 months before their scheduled surgery to explain the study timeline, which included a preoperative interview and 3 postoperative interviews ( 6 months, 12 months, and 24 months following surgery). Twenty-three patients consented and enrolled in the study.

This study was approved by the SickKids Research Ethics Board (\#1000025502).

\section{Data collection}

Between 2016 and 2021, 60-min semi-structured individual interviews were conducted with patients aged $16-20$ years across three timepoints following surgery: 6 months, 12 months, and 24 months. Interviews were conducted in-person or via Zoom by research coordinators trained in qualitative research methodology. The interview guide (Supplementary) was developed by team members ( $\mathrm{JH}$ and $\mathrm{AR}$ ) with clinical experience with adolescent bariatric surgery patients. Key questions prompted reflection on whether and how surgery has met their expectations for weight loss, physical function, social relations, mood and self-image, and support systems. All interviews were recorded, de-identified, and transcribed verbatim.

Parents/caregivers were not involved in the interviews, since our goal was to explore the adolescent perspective without input or potential influences from parents/caregivers, especially since some interview questions revolved around patients' thoughts on their perceived family support, environment, care, and other support systems.

\section{Analysis}

Transcripts were analyzed using Dedoose Version 8.3.45 [25]. A deductive thematic analysis framework was developed (ML and TS) and reviewed (MS and $\mathrm{JH}$ ). The analysis framework was informed by Nowell et. al.'s adaptation to Braun and Clarke's methodology [26, 27]. Transcripts were selected for analysis via purposive sampling to balance demographic characteristics, type of surgery, surgery date, and to minimize attrition across all three timepoints. The remaining transcripts were reviewed by investigators to ensure no new themes emerged. Transcripts were coded and analyzed until thematic saturation was reached. To ensure no new substantial themes emerged, transcripts were periodically assessed at each timepoint throughout analysis as per Guest et al. [28]. Two independent coders (ML and TS) adapted the coding framework concurrently to ensure inter-rater reliability (Cronbach's $a=0.84$ ) and maintain a consistent coding process while resolving conflicts through discussion. All reviewers discussed and refined final themes to ensure internal coherency and consistency. Member checking, a process of verifying the accuracy of final themes with participants was employed to improve reliability and rigor of our analysis $[29,30]$.

\section{RESULTS}

Saturation was reached after 15 interviews at 6 months, 15 at 12 months, and 15 at 24 months, representing 18 participants (5 male, 13 female) aged $17 \pm 1$ years, with mean percentage weight loss \pm SD at 6,12 , 24 months of $21.9 \% \pm 0.1,25.1 \% \pm 0.1$, and $27.0 \%$ \pm 0.2 , respectively (Table 1 ).

Five themes were identified related to: (1) weight-loss expectations vs. reality, (2) social landscape, (3) body image, (4) eating and moving, and (5) challenges for long-term success. Select quotations characteristic of dominant themes are presented in-text, with others in Table 2.

\section{Weight-loss: expectations vs. reality}

Participants distinguished between their hopes for weight loss and setting "realistic expectations" to avoid disappointment: "I'm not a person that sets expectation[s] in general...because I didn't wanna get let down, I couldn't imagine losing this much weight" (BS2-24M).

Expectation management varied between participants, with six participants losing more weight or losing weight faster than expected, seven participants experienced weight changes concordant with their expectations, and five reporting disappointment at 12 or 24 months. Some participants were surprised by the magnitude of their weight loss in the first few months following surgery, "it was only like 3 or 4 months in, I was just losing so much weight so quickly...I've lost like 100 pounds so it's still crazy to me." (BS2-24M).

Participants whose expectations for weight loss were unmet by the 12-month benchmark acknowledged that trajectories may differ for each individual. However, five participants who expressed disappointment with their outcome by 24 months attributed it to their surgery type. Although most were satisfied with their choice despite not fully achieving their desired outcome, two regretted their choice for the VSG over the RYGB, which they believed would have led to greater weight-loss: "I wish 
Table 1. Participant demographics.

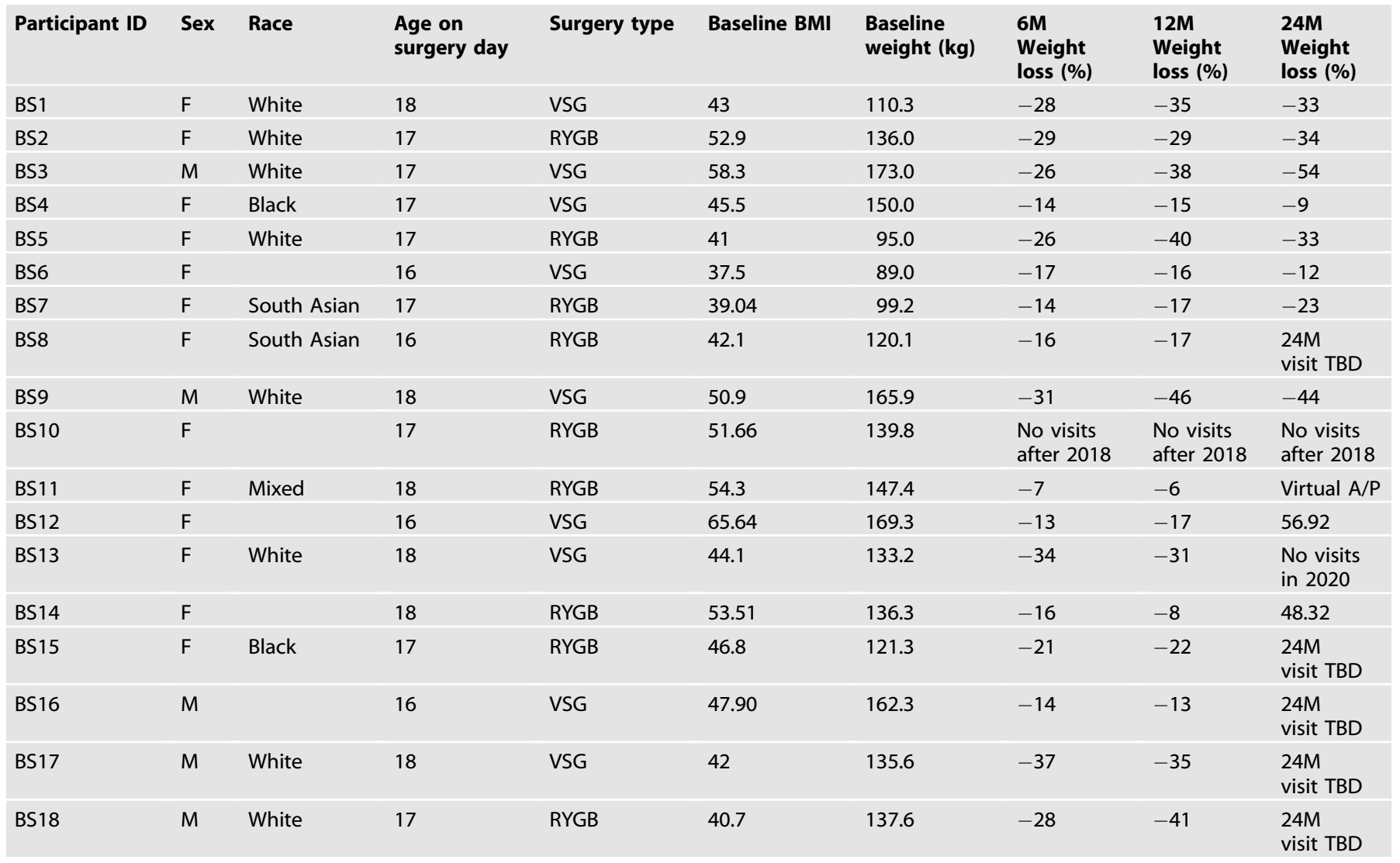

VSG sleeve gastrectomy, RYGB Roux-en-Y gastric bypass, $B M I$ body mass index, $A / P$ appointment, $T B D$ to be determined.

I'd done the bypass instead now... I'm pretty sure it's more intense and you're supposed to lose more weight...I'm still not seeing the kind of results I've seen [from] friends who did [the RYGB]" (BS4-24M).

\section{Social landscape}

Evolving social relationships. All participants described increased comfort engaging in social encounters and accessing new social opportunities, although they felt conflicted by the external validation they received implicitly (i.e., being seen or treated differently after surgery) or explicitly (i.e., comments).

Five participants described feeling more comfortable in public after surgery. This change was more pronounced in participants who moved to new environments (e.g., postsecondary education) shortly after surgery: "before, I definitely avoided a lot of situations because of my weight, like I was so scared to go to college because I was like 'I didn't know anybody, it would be hard for me to make friends', but now [I've]made friends so easily" (BS2-24M). Two participants reported receiving romantic attention and increased comfort pursuing romantic relationships: "I ran into this guy at a bus stop and then we started talking. Before, I would not have started talking to him... people were uncomfortable with who I was, [and] I wasn't comfortable either." (BS1-12M). This participant reaffirmed this belief at 24 months: "I'm in a relationship now. I was like super conscious about my body, but like we'll have sex and he's like 'you're so pretty,' and it makes me feel really nice." (BS1-24M).

Almost all participants recognized changes in attention and external perceptions as early as 6 months postoperatively, especially among those with whom they never previously connected. Participants also received explicit comments such as 'oh my gosh like you look so different', or 'I didn't recognize you' (BS18-6M). Some viewed this positively: "before surgery, because I was so overweight, I feel like people felt uncomfortable around me... afterwards, my relationships with people have gotten stronger in a sense, I feel more open towards them and I feel like they're more open towards me too" (BS6-6M). However, many participants were conflicted between embracing new social opportunities or rejecting them based on their belief that weight should not influence relationships: "I wouldn't have really wanted to have a friend just because I look better like this...it's kind of shallow. Obviously, I appreciate people are nicer, but [I'm] the same person" (BS4-6M). This dissonance between being treated differently while maintaining a consistent internal identity caused distress across all timepoints, with one participant finding the behavior "rude and very disrespectful" (BS12-6M).

All participants mentioned that most acquaintances were unaware of their surgery and often asked about their weight loss, but they shared information selectively and often disengaged to protect their mental health.

New and changing experiences. Almost all participants felt that surgery redefined clothes shopping as a social activity by reducing weight-related anxiety and allowing them to shop with their friends: "it's so much easier to find clothes [now] 'cuz I used to have anxiety when going clothes shopping... when you have the largest pants in the store but they don't fit you, it's like the worst feeling" (BS17-6M).

Most participants were apprehensive about substance use due to medical advice regarding health-related risks of alcohol consumption. Most participants abstained from substances: "before [surgery], I definitely would've drank, I would smoke weed...but they said you can't drink alcohol for a year or so [postoperatively] because it affects your body differently than before, 
Table 2. Representative quotes selected from participant interviews, assorted by themes and subthemes.

\section{Theme}

Weight-loss: expectations vs. reality

Social landscape

Body image

Eating \& moving

Challenges for long-term success

\section{Representative quotes}

- "it's been really quick. It's been a lot quicker than I expected. I know that in a lot of the information sessions they're like 'oh yeah for the sleeve it's a year and a half, two years, it's really longer than the bypass, but I just kind of dropped. It's very strange and it's kind of concerning, but it's just been insane" (BS1-6M)

- "I've heard from other patients and the doctors that [with] the bypass you lose more weight overall and it's harder for you to revert back to the state you were beforehand" (BS6-24M)

\section{Evolving social relations:}

- "I go out more with [my siblings] now, I'm not as scared...before surgery, I would just like hibernate in my room or stay home because I was always anxious... now I go more out in public and like do activities that I always wanted to do but I held myself back from" (BS11-6M)

- "it's sad that people view fat people with such hatred, and that now that I'm no longer fat, [it's] more acceptable to be my friend...it's such a shame that people are like 'oh you're so fat, I don't wanna talk to you', 'oh you've lost weight? I wanna talk to you now 'cause I think you're a cool person"' (BS1-6M)

\section{New and changing experiences:}

- "I was definitely very self-conscious about even like going to a store and buying clothes because like I wouldn't be able to fit in them...I would feel very bad about it, but now I can like go in and feel great" (BS6-12M)

- "I think [surgery] makes me not want to do it more. Like especially like drinking... because I'm more cautious about like how it's gonna affect my body, like my new 'stomach" (BS2-6M)

- "they're like 'you're gonna have so much loose skin', and I'm like 'I already have all this flab everywhere, like what's the difference!' and it's different. Having the skin hanging down opposed to being fat like it's just different, and I hate it, and I shoulda believed it" (BS1-6M)

- "even though it's improving a lot, it's still a scary image for me and I don't like the way that the extra skin looks at this moment" (BS10-12M)

- "my mom brought this up the other day [that] I always bring up if I look fatter, but she goes 'but before, when you were fat, you never asked if you were fat' but now I actually look at my stomach more to see if it's gone, if it has a little hump like I've start[ed] filling up fat again" (BS9-24M)

\section{Eating feels different:}

- "the feelings you get when you eat too much or eat the wrong thing...[the medical team] told me it was gonna happen and I was like 'Ya, ya, okay' but like, I didn't expect to feel it that much" (BS11-24M)

- "before my surgery, my favorite food was French fries but now I can't have like fried foods... some foods I didn't used to like before my surgery, now I eat them, for example Brussel sprouts, asparagus...it's definitely changed my palette, expanded it to more like fruits and vegetables" (BS12-6M)

- "I used to love like whipped cream and stuff. I cannot eat that anymore and I'm fine with it like it doesn't bother me. I'm like "oh if it's gunna make me sick, I don't want it"(BS2-24M)

\section{Physical mobility and function:}

- "I'm more inclined to move around than to just sit still and watch movies" (BS14-12M)

- "I'm able to do so much more activity... before I would like I wouldn't get out of breath from the small stuff, like I wouldn't be able to walk on the treadmill and now when I work out I can like go for a run...like simple stuff like getting down like sitting on the ground and standing up" (BS6-12M)

Life transitions: "my support system kinda just looks like my mom and dad just making sure uh like everything is available to me it is a little different 'cause I am at university so it's more of just me making sure I've kinda still following along, um but they're always kinda checkin' up on me making sure I'm doing what I need to do, taking vitamins stuff like that so support system is strong" (BS18-6M)

Lifelong journey: "no matter how scary it may seem, cus it is like a big life change but it does pay off and there will be obstacles but, there's obstacles are just that they're just obstacles they're not like stop signs" (BS6-24M)

Sources of support: "the support I've got from my friends and family. Not just my mom and dad, like my aunts and uncles have all been really supportive. And all my friends too, like they support me, and my friends always like 'oh make sure you're eating' and I definitely don't think l'd be as successful if it wasn't for them" (BS6-6M)

Advice for others:

- "it's hard work, you're gonna have to work your butt off, and it's gonna suck sometimes, but you're gonna get through it, and it's worth it." (BS 1-6)

- "really understand that it's not a quick fix, like it's not just a snap of the button and then hey! You'll never have weight problems again. It's a tool and a lot of people think it is gonna solve all their problems...there's a lot more work to it than that." (BS13-12)

- "there will be obstacles but they're just obstacles, they're not like stop signs... it's a lot of hard work and dedication so you have to really put your mind to it but if you're willing to do that, it will pay off" (BS 6-24M) so I'm staying away from that until the doctor says 'you can try it'."(BS6-6M). Six participants experimented with cannabis and alcohol, but emphasized that they exercised caution and avoided binge-drinking or behaviors they believed would pose significant health risks. This was mostly done in social settings, highlighting the role of substance use with peers.

\section{Body image}

Participants experienced positive impacts of surgery on their body image, confidence, and mood: "the way I look at myself, the way I carry myself, I just feel a lil bit more confident [now]" (BS18-6M). However, they consistently discussed balancing body image improvements with self-consciousness due to postoperative complications (e.g., excess skin, hair loss, awareness of body fat).

Most participants were aware of common side effects, although the extent of these experiences was unexpected. Pannus was a prominent one that was expected but generally unwelcome and was a concern at all timepoints: "since I've lost a ton of weight, I have a lot of loose skin...it's [been] so frustrating, because my body would look so good if I didn't have this loose skin" (BS2-24M). One 
participant discussed that although bigger bodies and stretch marks are becoming commonplace, excess saggy skin is not. In fact, this participant had a visceral reaction to their pannus: "it's on my body and I think it's gross... it's gross to feel, it's gross to look at" (BS 13-24 M). Consequently, three participants mentioned considering plastic surgery to reduce excess skin after weight stabilization.

All participants were warned of hair loss, but several were surprised by how quickly and to what degree this occurred: "they said 'you're gonna lose hair', and I'm like 'I [already] shed handfuls a day, I'm not gonna lose more hair', but it's just been coming out in bucket-loads." (BS1-6M). One participant mentioned the longlasting impacts at both 12- and 24 months: "I wasn't expecting that much [hair loss] and it's still not growing back" (BS7-24M).

\section{Eating and moving}

Eating feels different. Participants noted that surgery changed their eating routines, food choices, and food consciousness. All participants struggled with acute logistic adjustments in eating (i.e., smaller portions, more frequent intake, tracking nutrients) and attributed this difficulty to a mind-body disconnect: "it's your stomach that got surgery, so your brain doesn't know that your stomach is smaller so you will just eat like normal...but you have to control yourself" (BS12-6M). Dumping syndrome symptoms (i.e., nausea/vomiting, reflux, and stomach pain after eating) seemed most pronounced at 6 months and resolved with time as participants adjusted to new routines: "I didn't believe them at first... but there's no way to cheat, or else the dumping syndrome will be very very bad...I want[ed] to throw up all the time after I ate" (BS12-6M). Some noticed that their new eating routines reduced cravings, food-sneaking, mindless or binge-eating behaviors, and changed their relationship with food: "before, I didn't have any control over it, so l'd just keep eating...now because it's easier to tell when I'm full, I know when to stop." (BS6-6M).

Many participants also noted that surgery changed their palette and necessitated adaptation to new preferences and tolerances. It was noted that high-fat and high-sugar foods (e.g., pop, pizza, ice cream) which were previous favorites now made them sick, and that their palette expanded to include more fruits and vegetables. This was a welcomed change as they were invested in their health: "before [surgery], I used to hate broccoli, I couldn't even smell it, and I love [it] now...it feels good that I'm craving the healthy stuff and not the gross stuff" (BS11-24M).

Many participants felt that surgery made them more food conscious by enabling healthier, nutrition-based decisions, resisting temptations while grocery shopping, and associating guilt with junk food. Five participants also reported barriers to accessing healthy food, such as high cost, interpreting nutrition labels, and living on school campuses with limited food options and/or kitchen access: "being in college has made it harder...one day I went and got like a bunch of healthy food and it costed me \$17 for a small container of watermelon, cucumber rolls and a protein bar" (BS14-12M).

Approximately half the participants reported feeling distress explaining their reduced stomach capacity to family and friends, and experienced guilt about wasting money or not finishing food. One participant cited an example of compensatory behaviors to avoid disappointing others: "my grandma made me food [and] wanted me to eat it all, and I couldn't. I didn't want to tell her because she [would've felt] bad so I put the food into an empty Timbit bag and threw it out the balcony" (BS17-6M).

Physical mobility and function. Most participants reported feeling healthier overall due to weight loss and resolved or reduced medical comorbidities. The highlight for all participants was improved mobility and daily function, noted as early as 6 months postoperatively: "It's so much easier to walk, or like run, play sports...everything is just easier, even basic things, like putting your socks and shoes, shaving my legs" (BS 2-6M). This increased physical endurance was more pronounced by 12 months and more than half the participants re-engaged in physical activities (e.g., running, weightlifting, biking).

\section{Challenges for long-term success}

Life transitions. Participants felt that life changes (e.g., living independently, employment, post-secondary education) disrupted their ability to establish and maintain a consistent postoperative routine. Many recognized the need for flexibility while adapting to new environments and finding new financial and social supports: "I've already had so many changes, like I've already got my first jobs, I was going away to school. There's always been so much, that adding surgery on top of it, it was just like 'okay! I just need to take a breath! You can deal with it" (BS1-6M). For participants undergoing postoperative management during the COVID-19 pandemic, the lack of routine has been challenging: "[pre-]COVID, I used to have a trainer that came to my house on a weekly basis, we'd do like kick boxing, cardio stuff....[but now] I go on my walks. That's basically all" (BS17-24M).

Lifelong journey. All participants recognized by 24 months that bariatric surgery is a lifelong journey requiring perseverance and commitment for long-term success: "it is like a big life change, it does pay off...there will be obstacles...but they're just obstacles, they're not like stop signs" (BS6-24M). Most participants were anxious and fearful of regaining weight. One participant described weight maintenance as a lifelong battle: "It's sort of got me like 'am I gonna be fighting this weight for the rest of my life?"' (BS13-24M). Following medical instructions (i.e., vitamin supplements, eating routines) postoperatively proved challenging for participants: "I didn't do exactly what the doctor told me to do...now I still [don't] but I know I should" (BS8-24M). Similarly, another participant talked about the importance of mental wellness: "[Although] I lost the weight, at the end of the day [I]realized...I wasn't super happy...My weight loss journey was everyone telling me to lose weight...I'm realizing I was happy when I was overweight...so, I'm just kinda learning to accept the change." (BS15-24M). Mental health conditions factored into the long-term success of our patients as they often influenced patients' satisfaction with postoperative outcomes, perceptions of evolving body image, and acceptance of suboptimal weight loss (e.g., weight regain, reduced weight loss, etc.).

Sources of support. The importance of both external (family, friends, healthcare team) and internal (self-motivation) sources of support was highlighted across all interviews. Overwhelmingly, participants appreciated the support they received from family and friends in regaining stability postoperatively and saw this support as a necessity in achieving sustainable long-term goals. However, one participant grappled with micromanagement: "it can sometimes be too much of like 'you need to be eating'...l like that they're so caring, but at the same time I'm just like 'back off a little bit"' (BS1-6M).

Participants appreciated structured postoperative care and guidelines provided by the healthcare team: "I really look forward to the weekly dietitian visits because there's always a new challenge, whether it's a new holiday or me going away for a week... [it'd] be so hard to go through that...I wouldn't know how to handle any of it" (BS6-6M). However, as mentioned above, the pandemic has impacted many participants' routines, including their ability to regularly attend surgery support groups.

Participants provided advice for other adolescents considering bariatric surgery, emphasizing making an informed, autonomous decision, setting realistic expectations for outcomes, and being ready for change. Although some participants did not follow medical recommendations (e.g., vitamin supplements), they suggested future participants should avoid non-adherence. 
Participants also discussed the importance of focusing on individual journeys and avoiding comparison: "don't focus too much on the numbers...take it at your own pace, follow your own path...do what you need to do, and success will come" (BS11-12M).

\section{DISCUSSION}

This qualitative study followed the perspectives of 18 adolescent bariatric surgery patients at 6,12 , and 24 months postoperatively. The predominant themes identified related to (1) weight-loss expectations vs. reality, (2) social landscape, (3) body image, (4) eating and moving, and (5) challenges for long-term success. Adolescents were primarily positive or ambivalent about their personal experiences following bariatric surgery.

To our knowledge, studies following adolescence after bariatric surgery have been reported from the United States [17], Europe $[2,13,18,19,31,32]$, and Qatar [33]. Most reports were crosssectional, with some including one-year follow-up. Accounting for differences in geography and healthcare systems, study methodology, and number of participants assessed, our findings build on existing literature.

Longitudinal follow-up of participants enabled temporal tracking of participants' perspectives. There was significant focus on weight-loss benchmarks and physical adjustments (e.g., dumping syndrome) at 6 months. By 12 and 24 months, acute distress due to physical changes resolved for most participants, although many increasingly reported challenges of maintaining routines due to significant life transitions. Post-operative support from our interprofessional team was critical to address internal and external challenges to sustaining daily routines. Examples include patients working closely with our dietitians to develop self-management strategies for problematic eating behaviors, and attending surgery support group sessions for social support and peer advice on adhering to postoperative guidance. Patients also recognized the need to develop self-management skills to normalize setbacks and continue working towards their goals, aligning with recent literature on the importance of embracing self-management for long-term success [34].

Similar to other reports, most adolescent patients felt increased body comfort and physical mobility, improved confidence, and greater capacity to pursue other endeavors (e.g., part-/full-time employment and post-secondary education) after bariatric surgery $[12,13,17]$. Although most of our participants reported improved body image and confidence, some struggled to reconcile their internal identity with evolving external perceptions and expressed discomfort with new attention to their weight loss. Thus, it is important to provide peri- and postoperative support on body image and the possible impact on social relationships.

Consistent with our findings, three studies reported that body dissatisfaction is common among those with unrealistic weightloss goals, which cannot be achieved by bariatric surgery alone $[18,35,36]$. In contrast, participants achieving better postoperative outcomes endorsed realistic pre-surgical expectations [13, 27]. Although there were numerous positive experiences, some participants in our study offered a nuanced outlook on their weight loss. A novel observation includes participants being discouraged by the slowdown of weight loss by 24 months and worry about weight regain. These findings demonstrate the need for expectation management for weight loss and associated nutritional needs.

All other short-term studies recognized that adolescent patients struggled and experienced the loss of follow-up as they transitioned to adult healthcare. Childerhose et al. and Nordin et al. suggest that surgery timing is an important factor in optimizing postoperative outcomes $[17,19]$. Our participants did not share this experience, perhaps attributable to our continued 2-year STOMP postoperative follow-up before transitioning to adult care. However, they reported challenges with other life transitions (e.g., moving out, employment, education) and emerging independence (e.g., financial/food security, kitchen access, transportation). Personalized counseling to help adolescents better prepare for the impact of life transitions postbariatric surgery is an important consideration during this unique developmental period.

Literature suggests lower alcohol use rates in adolescent bariatric surgery patients due to sensitization, although those who did consume alcohol were more likely to binge-drink due to increased socialization and replacement of food with alcohol as a coping mechanism [37]. In our cohort, higher-risk behaviors such as excessive substance use were not highlighted, as they mostly reported cautious, responsible use or abstinence from substances. The underreporting of these behaviors could be due to selfreporting bias.

Research suggests these patients are more likely to engage in high-risk sexual activity due to a lack of preparedness, reporting higher-than-anticipated pregnancy rates in an adolescent bariatric surgery cohort $[16,38]$. In our cohort, a few participants disclosed increased sexual activity or new romantic relationships. These findings, while limited, demonstrate a need for proactive patient education on safe sexual practices.

This study fills a research gap in the adolescent population, which lags behind adult bariatric surgery literature. In contrast to qualitative studies in adult studies, this study highlights several unique features of the adolescent bariatric population. As mentioned above, the transition from adolescence to early adulthood is a challenging period to undergo bariatric surgery, as life transitions (i.e., increased autonomy, evolving social support, financial independence) pose unique disruptions to weight maintenance and consistent postoperative routines. These factors coalesce to impact their social interactions with peers, family members, sexual partners, etc. As a result of their changing social landscape, they may experience limited control over their weight maintenance routines compared to adults [39]. The transition from pediatric to adult healthcare during the early postoperative period, leading to patients feeling "lost in the system", is also a unique challenge for this population [11, 19].

A strength of this study is limited selection bias, as the majority of patients who underwent bariatric surgery at our institution participated in this study, irrespective of outcome satisfaction. Furthermore, participants were diverse in terms of ethnic background, sex, and socioeconomic status. Two-year postoperative follow-up also minimized limitations cited by other studies including loss to follow-up as patients transitioned to adult healthcare, and allowed us to capture temporal trends in qualitative outcomes. This practice aligns with evidence demonstrating benefits of a structured transition program [19, 40].

Our findings may be limited by social desirability bias due to the self-report nature of our interviews. Consequently, higher-risk or less adherent behaviors may be underreported. Furthermore, the COVID-19 pandemic complicated postoperative care, follow-up frequency, and ability to maintain postoperative routines. For example, Athanasiadis et al. identified that postoperative bariatric patients regained some weight during the COVID-19 pandemic due to more snacking, more anxiety, and less engagement with aerobic exercise [41].

An area for future exploration includes the perspectives of healthcare providers and caregivers. These additional insights will contribute to a holistic view of the adolescent bariatric surgery process and further strengthen perioperative support for these patients.

In summary, this study contributes to the growing body of research on postoperative experiences of adolescent bariatric surgery patients over a 2-year period. Important implications for clinical practice include discussing the timing of surgery with future participants to ensure they are well-informed, and managing expectations in the context of important life transitions 
throughout the formative years of adolescence. Our participants valued ongoing support from the healthcare team after surgery, thus a delayed transition of care postoperatively after 18 years of age is likely beneficial given the known challenges youth face in transitioning from youth to adult care [19, 40]. Furthermore, programs should provide clear, realistic expectations for weight loss and anticipated changes in eating to minimize disappointment or body dysmorphia. For patients who have slow weight loss, close examination of contributory factors should be explored early by the healthcare team. Similarly, our study highlights the complexities of social interactions following weight loss. Anticipatory guidance may help teens navigate these relationships.

\section{REFERENCES}

1. Pratt JSA, Browne A, Browne NT, Bruzoni M, Cohen M, Desai A, et al. ASMBS pediatric metabolic and bariatric surgery guidelines, 2018. Surg Obes Relat Dis. 2018;14:882-901.

2. Paulus GF, de Vaan LEG, Verdam FJ, Bouvy ND, Ambergen TAW, van Heurn LWE. Bariatric surgery in morbidly obese adolescents: a systematic review and metaanalysis. Obes Surg. 2015;25:860-78.

3. Armstrong SC, Bolling CF, Michalsky MP, Reichard KW. Pediatric metabolic and bariatric surgery: evidence, barriers, andbest practices. Pediatrics. 2019;114. https://pubmed.ncbi.nlm.nih.gov/31656225/.

4. Durkin N, Desai AP. What Is the evidence for paediatric/adolescent bariatric surgery? Curr Obes Rep. 2017;6:278-85.

5. Inge TH, Jenkins TM, Xanthakos SA, Dixon JB, Daniels SR, Zeller MH, et al. Longterm outcomes of bariatric surgery in adolescents with severe obesity (FABS-5+): a prospective follow-up analysis. Lancet Diabetes Endocrinol. 2017;5:165-73.

6. Inge TH, Courcoulas AP, Jenkins TM, Michalsky MP, Helmrath MA, Brandt ML, et al. Weight loss and health status 3 years after bariatric surgery in adolescents. N Engl J Med. 2016:374:113-23.

7. Inge TH, Courcoulas AP, Jenkins TM, Michalsky MP, Brandt ML, Xanthakos SA, et al. Five-year outcomes of gastric bypass in adolescents as compared with adults. N Engl J Med. 2019;380:2136-45.

8. Steinbeck KS, Lister NB, Gow ML, Baur LA. Treatment of adolescent obesity. Nat Rev Endocrinol. 2018;14:331-44.

9. Sweeting H, Smith E, Neary J, Wright C. 'Now I care': a qualitative study of how overweight adolescents managed their weight in the transition to adulthood. BMJ open. 2016;6:e010774.

10. Gray WN, Schaefer MR, Resmini-Rawlinson A, Wagoner ST. Barriers to transition from pediatric to adult care: a systematic review. J Pediatr Psychol. 2018;43:488-502.

11. Shrewsbury VA, Baur LA, Nguyen B, Steinbeck KS. Transition to adult care in adolescent obesity: a systematic review and why it is a neglected topic. Int J Obes. 2014;38:475-9.

12. Trooboff SW, Stucke RS, Riblet NB, Kulkarni AS, Anand R, Casey A, et al. Psychosocial outcomes following adolescent metabolic and bariatric surgery: a systematic review and meta-analysis. Obes Surg. 2019;29:3653-64.

13. Poulsen L, Klassen A, Jhanwar S, Pusic A, Roessler KK, Rose M, et al. Patient expectations of bariatric and body contouring surgery. Plast Reconstr Surg Glob Open. 2016;4:e694.

14. Hillstrom KA, Graves JK. A review of depression and quality of life outcomes in adolescents post bariatric surgery. J Child Adolesc Psychiatr Nurs. 2015;28:50-9.

15. White B, Doyle J, Colville S, Nicholls D, Viner RM, Christie D. Systematic review of psychological and social outcomes of adolescents undergoing bariatric surgery, and predictors of success. Clin Obes. 2015;5:312-24.

16. Zeller MH, Pendery EC, Reiter-Purtill J, Hunsaker SL, Jenkins TM, Helmrath MA, et al. From adolescence to young adulthood: trajectories of psychosocial health following Roux-en-Y gastric bypass. Surg Obes Relat Dis. 2017;13:1196-203.

17. Childerhose JE, Eneli I, Steele KE. Adolescent bariatric surgery: a qualitative exploratory study of US patient perspectives. Clin Obes. 2018;8:345-54.

18. Doyle J, Colville S, Brown P, Christie D. How adolescents decide on bariatric surgery: an interpretative phenomenological analysis. Clin Obes. 2018; 8:114-21.

19. Nordin K, Brorsson A-L, Ekbom K. Adolescents' experiences of obesity surgery: a qualitative study. Surg Obes Relat Dis. 2018;14:1157-62.

20. Coulman KD, MacKichan F, Blazeby JM, Owen-Smith A. Patient experiences of outcomes of bariatric surgery: a systematic review and qualitative synthesis. Obes Rev. 2017;18:547-59.

21. Lynch Al, McGowan E, Zalesin KC. "Take Me through the History of Your Weight": Using qualitative interviews to create personalized weight trajectories to understand the development of obesity in patients preparing for bariatric surgery. J Acad Nutr Diet. 2018;118:1644-54.
22. Li MK, Regina A, Strom M, Kim MS, Philipp-Muller N, Hamilton JK. "It's a tool, not a cure": the preoperative teen perspective on bariatric surgery. Surg Obes Relat Dis. 2021;11:1190-7.

23. Walsh Ó, Dettmer E, Regina A, Ye L, Christian J, Hamilton J, et al. "I don't want them to think that what they said matters": how treatment - seeking adolescents with severe obesity cope with weight-based victimization. Clin Obes. 2021;11: e12437.

24. Ontario Bariatric Network. Managing the bariatric surgery patient: a practical reference guide for primary care providers in Ontario. 2021. https://www. corecarefht.ca/PCPReferenceGuide.pdf.

25. Dedoose Version 8.3.45, web application for managing, analyzing, and presenting qualitative and mixed method research data. Los Angeles, CA: SocioCultural Research Consultants, LLC; 2020.

26. Nowell LS, Norris JM, White DE, Moules NJ. Thematic analysis: striving to meet the trustworthiness criteria. Int J Qual Methods. 2017;16:1609406917733847.

27. Braun V, Clarke V. Using thematic analysis in psychology. Qual Res Psychol. 2006:3:77-101.

28. Guest G, Bunce A, Johnson L. How many interviews are enough? An experiment with data saturation and variability. Field methods. 2016;18:59-82.

29. Lincoln YS, Inquiry GN. The Blackwell encyclopedia of sociology. Beverly Hills, Calif: Sage. 1985:416..

30. Lewis J. Redefining qualitative methods: believability in the fifth moment. Int J Qual Methods. 2009;8:1-14.

31. Herget S, Rudolph A, Hilbert A, Blüher S. Psychosocial status and mental health in adolescents before and after bariatric surgery: a systematic literature review. Obes Facts. 2014;7:233-45.

32. Silberhumer GR, Miller K, Pump A, Kriwanek S, Widhalm K, Gyoeri G, et al. Longterm results after laparoscopic adjustable gastric banding in adolescent patients: follow-up of the Austrian experience. Surg Endosc. 2011;25:2993-9.

33. El-Matbouly MA, Khidir N, Touny HA, El Ansari W, Al-Kuwari M, Bashah M. A 5-year follow-up study of laparoscopic sleeve gastrectomy among morbidly obese adolescents: does it improve body image and prevent and treat diabetes? Obes Surg. 2018;28:513-9.

34. Youssef A, Mylopoulos M, Maunder R, Wiljer D, Cassin SE, Wnuk S, et al. Understanding bariatric patients' experiences of self-management post-surgery: a qualitative study. [published online ahead of print, 2021 Jun 14]. Clin Obes. 2021; e12473. https://doi.org/10.1111/cob.12473.

35. Ratcliff MB, Eshleman KE, Reiter-Purtill J, Zeller MH. Prospective changes in body image dissatisfaction among adolescent bariatric patients: the importance of body size estimation. Surg Obes Relat Dis. 2012;8:470-5.

36. Graham Y, Hayes C, Small PK, Mahawar K, Ling J. Patient experiences of adjusting to life in the first 2 years after bariatric surgery: a qualitative study. Clin Obes. 2017;7:323-35.

37. Spadola CE, Wagner EF, Dillon FR, Trepka MJ, Cruz-Munoz NDL, Messiah SE. Alcohol and drug use among postoperative bariatric patients: a systematic review of the emerging research and its implications. Alcohol Clin Exp Res. 2015;39:1582-601.

38. Zeller MH, Brown JL, Reiter-Purtill J, Sarwer DB, Black L, Jenkins TM, et al. Sexual behaviors, risks, and sexual health outcomes for adolescent females following bariatric surgery. Surg Obes Relat Dis. 2019;15:969-78.

39. Hsia DS, Fallon SC, Brandt ML. Adolescent bariatric surgery. Arch Pediatr Adolesc Med. 2012;166:757-66.

40. Sheehan AM, While AE, Coyne I. The experiences and impact of transition from child to adult healthcare services for young people with Type 1 diabetes: a systematic review. Diabet Med J Br Diabet Assoc. 2015;32:440-58.

41. Athanasiadis DI, Hernandez E, Hilgendorf W, Roper A, Embry M, Selzer D, et al. How are bariatric patients coping during the coronavirus disease 2019 (COVID19) pandemic? Analysis of factors known to cause weight regain among postoperative bariatric patients. Surg Obes Relat Dis. 2021;17:756-64.

\section{ACKNOWLEDGEMENTS}

We would like to acknowledge our patients for their openness and willingness to participate in this research. We are additionally grateful to Kristina Cordeiro and Jessica Jeong for their contributions to earlier stages of the research, and to Preeti Grewal and Elizabeth Dettmer for their ongoing involvement and insights.

\section{AUTHOR CONTRIBUTIONS}

AR, AT, MS, and JH contributed significantly to the study's conception and design and data collection. ML and TS led the data analysis, interpretation, and manuscript preparation with input from all authors. All authors contributed to editing and revising the manuscript. All authors have read and approved the final manuscript. 


\section{COMPETING INTERESTS}

$\mathrm{JH}$ is supported by the SickKids University of Toronto Mead Johnson Chair in Nutritional Science, which provides unrestricted research funds. The remaining authors declare no competing financial interests.

\section{ADDITIONAL INFORMATION}

Supplementary information The online version contains supplementary material available at https://doi.org/10.1038/s41366-021-00928-w.
Correspondence and requests for materials should be addressed to J.H.

Reprints and permission information is available at http://www.nature.com/ reprints

Publisher's note Springer Nature remains neutral with regard to jurisdictional claims in published maps and institutional affiliations. 